\title{
ALMOST CONTACT STRUCTURES AND CURVATURE TENSORS
}

\author{
By DiRK Janssens ANd Lieven Vanhecke
}

\begin{abstract}
We determine an orthogonal decomposition of the vector space of some curvature tensors on a co-Hermitian real vector space, in irreducible components with respect to the natural induced representation of $U(n) \times 1$. One of the components is used to introduce a Bochner curvature tensor on a class of almost co-Hermitian manifolds (or almost contact metric manifolds), called $C(\alpha)$-manifolds, containing e.g. co-Kählerian, Sasakian and Kenmotsu manifolds. Other applications of the decomposition are given.
\end{abstract}

\section{Introduction.}

In his study on Betti numbers of Kähler manifolds, S. Bochner introduced a tensor which had to take over in his theory the role of the Weyl tensor on Riemannian manifolds. More precisely, a conformally flat manifold was considered as an extension of a real space form. So, a Bochner flat Kähler manifold had to be an extension in the same sense of a complex space form.

The Weyl tensor is well known as a conformal invariant of Riemannian manifolds but the Bochner tensor on Kähler manifolds was defined in a completely formal way. Several attempts were made to find a geometrical interpretation.

Besides Kähler manifolds and the more general almost Hermitian manifolds, one has also studied classes of odd-dimensional manifolds with additional structures, namely the almost co-Hermitian manifolds or almost contact metric manifolds; in particular Sasakian, co-Kählerian and Kenmotsu manifolds.

A natural problem arises here: Is it possible to construct a "Bochner curvature tensor" for these classes of manifolds? A nice way to introduce this tensor is to use decomposition theory of spaces of curvature tensors. Singer and Thorpe obtained in this way the Weyl tensor [20] (see also [17]) and the Bochner tensor was derived with the Hermitian version of this decomposition in [16], [21]. Proceeding in the same way, the second author defined a Bochner tensor for $\mathrm{AH}_{3}$-manifolds (a class of almost Hermitian manifolds containing e.g. the nearly Kähler manifolds) [24], [27]. The general case is given completely in [34].

In this paper we define with a decomposition theory a Bochner curvature

Received October, 18, 1979 
tensor for a class of almost contact metric manifolds. The elements of this class are called almost $C(\alpha)$-manifolds and are introduced in section 2. They are defined by a condition on the Riemann curvature tensor. Examples are Sasakian and co-Kählerian manifolds, and also the manifolds studied by Kenmotsu [12]. In sections 3 and 4 we develop the algebraic theory of $C$-curvature tensors on co-Hermitian vector spaces and prove the decomposition theorem. In section 5 we give a complete algebraic characterization of the orthogonal decomposition by considering the naturally induced representation of $U(n) \times 1$ in the space of $C$-curvature tensors. The components of this decomposition are the irreducible components of this representation. Geometrical applications are given in section 6 and in section 7 we derive some useful inequalities relating the norm of the curvature tensor, the norm of the associated Ricci tensor and the scalar curvature. Finally we study in section 8 the decomposition of proper generalized $C(\alpha)$-curvature tensor fields (i. e. $C(\alpha)$-curvature tensor fields satisfying the second Bianchi identity) on Sasakian manifolds. As for Riemannian and Kähler manifolds [17], [16], the Codazzi equation for the Ricci tensor will play an important role.

We note that the Bochner tensor obtained in this paper for the class of Sasakian manifolds is just the $C$-Bochner tensor introduced by Matsumoto and Chūman [15]. C(0)-curvature tensor fields on Sasakian manifolds are the $S$-curvature like tensor fields introduced in [33].

\section{Almost $C(\alpha)$-manifolds.}

Let $M$ be a real $(2 n+1)$-dimensional $C^{\infty}$ manifold and $\mathscr{X}(M)$ the Lie algebra of $C^{\infty}$ vector fields on $M$. An almost co-complex structure on $M$ is defined by a $C^{\infty}(1,1)$-tensor field $\varphi$, a $C^{\infty}$ vector field $\xi$ and a $C^{\infty} 1$-form $\eta$ on $M$ such that for any point $x \in M$ we have

$$
\begin{gathered}
\varphi_{x}^{2}=-I+\eta_{x} \otimes \xi_{x}, \\
\eta_{x}\left(\xi_{x}\right)=1,
\end{gathered}
$$

where $I$ denotes the identity transformation of the tangent space $T_{x} M$ at $x$. Manifolds equipped with an almost cocomplex structure are called almost cocomplex or almost contact manufolds. A Riemannian manifold $M$ with metric tensor $g$ and with an almost cocomplex structure $\left(\varphi, \xi, \gamma_{i}\right)$ such that

$$
\forall X, Y \in \mathcal{X}(M): g(\varphi X, \circ Y)=g(X, Y)-\eta(X) \eta(Y)
$$

is an almost co-Hermitzan or an almost contact metruc manfold. The existence of an almost cocomplex structure on $M$ is equivalent with the existence of a reduction of the structural group to $U(n) \times 1$, i. e. all the matrices of $O(2 n+1)$ of the form

$$
\left(\begin{array}{rrr}
A & B & 0 \\
-B & A & 0 \\
0 & 0 & 1
\end{array}\right)
$$


where $A$ and $B$ are real $(n, n)$-matrices.

The Sasaki form (or fundamental 2 -form) $\Phi$ of an almost co-Hermitian manifold $(M, g, \varphi, \xi, \eta)$ is defined by

$$
\Phi(X, Y)=g(X, \varphi Y)
$$

for all $X, Y \in \mathscr{X}(M)$ and this form satisfies $\eta \wedge \Phi^{n} \neq 0$. This means that every almost co-Hermitian manifold is orientable and that $(\eta, \Phi)$ defines an almost cosymplectuc structure on $M$. If this associated structure is cosymplectic $(d \Phi=$ $d \eta=0), M$ is called an almost co-Kähler manrfold. When $\Phi=d \eta$, the associated almost cosymplectic structure is a contact structure and $M$ is an almost Sasakıan manifold. It is known that conversely every contact manfold has an almost Sasakian structure.

The Nijenhuis tensor of the $(1,1)$-tensor field $\varphi$ is the $(1,2)$-tensor field $[\varphi, \varphi]$ defined by

$$
[\varphi, \varphi](X, Y)=[\varphi X, \varphi Y]-[X, Y]-\varphi[X, \varphi Y]-\varphi[\varphi X, Y],
$$

where $[X, Y]$ is the Lie bracket of $X, Y \in \mathscr{X}(M)$. An almost cocomplex structure is called integrable if $[\varphi, \varphi]=0$ and normal if $[\varphi, \varphi]+2 d \eta \otimes \xi=0$. An integrable almost cocomplex structure is a cocomplex structure. A co-Kähler manfold (or normal cosymplectic manifold) is an integrable (or equivalently, a normal) almost coKähler manifold, while a Sasakıan manıfold is a normal almost Sasakian manifold.

For an extensive study of these manifolds we refer to [4], [6] and [19] where many examples are given. On the other hand, Kenmotsu studied in [12] another class of almost co-Hermitian manifolds, defined by the following conditions on the associated almost cosymplectic structure:

$$
\begin{gathered}
d \eta=0, \\
(d \Phi)(X, Y, Z)=\frac{2}{3} \underset{X, Y, Z}{\subseteq}\{\eta(X) \Phi(Y, Z)\},
\end{gathered}
$$

where $\subseteq$ denotes the cyclic sum. We call such manifolds almosi Kenmoisu manifolds. A normal almost Kenmotsu manifold is a Kenmotsu mamfold. The warped product $\boldsymbol{R} \times{ }_{f} F$ ( $F$ being a Kähler manifold and $f(t)=c e^{t}, c \in \boldsymbol{R}_{0}^{+}$) provides an example [5], [12].

The classes of co-Kähler, Sasakian and Kenmotsu manifolds are precısely the three classes which occur in a classification theorem of connected almost coHermitian manifolds $M^{2 n+1}$ for which the automorphism group has maximal dimension $(n+1)^{2}$ (see [22]).

The Riemannian connections $\nabla$ of Sasakian, co-Kähler and Kenmotsu manifolds have some well known properties which allow us to characterize these manifolds.

Theorem 2.1. Let $(M, g, \varphi, \xi, \eta)$ be an almost co-Hermitian manfold with Riemannian connection $\nabla$. Then

(i) $M$ is co-Kählerian if and only if $\nabla \varphi=0$; 
(ii) $M$ is Sasakian if and only if

$$
\forall X, Y \in \mathscr{X}(M):\left(\nabla_{X} \varphi\right) Y=g(X, Y) \xi-\eta(Y) X ;
$$

(iii) $M$ is a Kenmotsu manıfold if and only if

$$
\forall X, Y \in \mathfrak{X}(M):\left(\nabla_{X} \varphi\right) Y=g(\varphi X, Y) \xi-\eta(Y) \varphi X .
$$

Kenmotsu used (iii) for the definition of his class of manifolds [12]. Furthermore we have

THEOREM 2.2. $\xi$ is a Killing vector field for co-Kähler and Sasakı manifolds, i.e.

$$
\forall X, Y \in \mathfrak{X}(M): g\left(\nabla_{X} \xi, Y\right)+g\left(X, \nabla_{Y} \xi\right)=0,
$$

while for Kenmotsu manifolds we have

$$
\forall X, Y \in \mathfrak{X}(M): g\left(\nabla_{X} \xi, Y\right)-g\left(X, \nabla_{Y} \xi\right)=0 .
$$

Finally, the Riemann curvature tensor $R$ on these manifolds satisfies some interesting identities which will play an important role in the decomposition theory.

THEOREM 2.3. Let $R$ be the Riemann curvature tensor on $M$ and $X, Y, Z, W$ $\in \mathscr{X}(M)$. We have

(i) for $M$ co-Kählerian:

$$
R(X, Y, Z, W)=R(X, Y, \varphi Z, \varphi W) ;
$$

(ii) for M Sasakıan:

$$
\begin{aligned}
R(X, Y, Z, W)= & R(X, Y, \varphi Z, \varphi W)-g(X, Z) g(Y, W)+g(X, W) g(Y, Z) \\
& +g(X, \varphi Z) g(Y, \varphi W)-g(X, \varphi W) g(Y, \varphi Z) ;
\end{aligned}
$$

(iii) for a Kenmotsu manrfold $M$ :

$$
\begin{aligned}
R(X, Y, Z, W)= & R(X, Y, \varphi Z, \varphi W)+g(X, Z) g(Y, W)-g(X, W) g(Y, Z) \\
& -g(X, \varphi Z) g(Y, \varphi W)+g(X, \varphi W) g(Y, \varphi Z) .
\end{aligned}
$$

With this theorem in mind we give

Definition 2.4. An almost $C(\alpha)$-manufold $M$ is an almost co-Hermitian manifold such that the Riemann curvature tensor satisfies the following property:

$\exists \alpha \in \boldsymbol{R}$ such that for all $X, Y, Z, W \in \mathscr{X}(M)$ :

$$
\begin{aligned}
R(X, Y, Z, W)= & R(X, Y, \varphi Z, \varphi W)+\alpha\{-g(X, Z) g(Y, W)+g(X, W) g(Y, Z) \\
& +g(X, \varphi Z) g(Y, \varphi W)-g(X, \varphi W) g(Y, \varphi Z)\} .
\end{aligned}
$$


A normal almost $C(\alpha)$-manifold is a $C(\alpha)$-manifold.

Co-Kählerian, Sasakian and Kenmotsu manifolds are respectively $C(0)$-, $C(1)$ and $C(-1)$-manifolds. We give now some examples for other values of $\alpha$.

Definition 2.5. An almost $\alpha$-Sasakıan manıfold $M$ is an almost co-Hermitian manifold such that $\Phi=\frac{1}{\alpha} d \eta, \alpha \in \boldsymbol{R}_{0} . \quad M$ is an $\alpha$-Sasakıan manıfold if the structure is normal.

Just as for Sasakian manifolds [4] one can prove

\section{THEOREM 2.6 .}

(i) An almost co-Hermitian manıfold $M$ is $\alpha$-Sasakian if and only if for all $X, Y \in \mathscr{X}(M)$

$$
\left(\nabla_{X} \varphi\right) Y=\alpha\{g(X, Y) \xi-\eta(Y) X\} .
$$

(ii) If $M$ is $\alpha$-Sasakıan, then $\xi$ is a Killing vector field and

$$
\nabla_{X} \xi=-\alpha \varphi X
$$

for all $X \in \mathfrak{X}(M)$.

(iii) An $\alpha$-Sasakian manifold is a $C\left(\alpha^{2}\right)$-manıfold.

If $M$ is a Sasakian manifold, then by a so-called $D$-homothetic transformation one can modify the Sasakian structure into an $\alpha$-Sasakian structure for $\alpha>0$. A $D$-homothetic transformation is a transformation of the original metric $g$ into a new Riemannian metric $g^{\prime}$ defined as follows:

$$
g^{\prime}(X, Y)=\frac{1}{\alpha} g(-X+\eta(X) \xi,-Y+\eta(Y) \xi)+\eta(X) \eta(Y)
$$

for all $X, Y \in T_{x} M$ and all $x \in M$. The two metrics are homothetic when restricted to the distribution $\eta=0$. On the other hand $(M, g, \varphi,-\xi,-\eta)$ is a $(-1)$-Sasakian structure if $(M, g, \varphi, \xi, \eta)$ is Sasakian. Starting with the $(-1)$ Sasakian structure one constructs $\alpha$-Sasakian structures for $\alpha<0$. We note that $\alpha$-Sasakian manifolds are quasi-Sasakian [3]. They provide examples of $C(\lambda)$ manifolds with $\lambda>0$.

To obtain examples for $\lambda<0$, we use the same procedure but starting now with Kenmotsu manifolds.

Definition 2.7. An almost $\alpha$-Kenmotsu manıfold is an almost co-Hermitian manifold such that

$$
\begin{gathered}
d \eta=0 \\
(d \Phi)(X, Y, Z)=\frac{2}{3} \alpha_{X, Y, Z}\{\eta(X) \Phi(Y, Z)\},
\end{gathered}
$$


for $\alpha \in \boldsymbol{R}_{0}$ and all $X, Y, Z \in \mathscr{X}(M) . \quad M$ is an $\alpha$-Kenmotsu manıfold if the structure is normal.

Here we have

THEOREM 2.8.

(i) An almost co-Hermitian manifold is an $\alpha$-Kenmotsu manifold is and only if

$$
\left(\nabla_{X} \varphi\right) Y=\alpha\{g(\varphi X, Y) \xi-\eta(Y) \varphi X\}
$$

where $X, Y \in \mathscr{X}(M)$.

(ii) An $\alpha$-Kenmotsu manıfold is a $C\left(-\alpha^{2}\right)$-manıfold.

Using the $D$-homothetic transformation defined by

$$
g^{\prime}(X, Y)=\alpha\{g(X, Y)-\eta(X) \eta(Y)\}, \quad \alpha>0,
$$

on a Kenmotsu manifold, one obtains an $\alpha$-Kenmotsu manifold with $\alpha>0$. By starting with $(M, g,-\varphi,-\xi,-\eta)$ we obtain a $(-\alpha)$-Kenmotsu manifold with $-\alpha<0$.

It is just for the class of almost $C(\alpha)$-manifolds that we shall define a Bochner curvature tensor. Therefore we develop a decomposition theory on an appropriate space of curvature tensors over a co-Hermitian vector space. This purely algebraic theory will give later on more information about important special classes of almost $C(\alpha)$-manifolds.

\section{3. $C(\alpha)$-curvature tensors.}

Let $V$ be an $n$-dimensional real vector space with inner product $g$. A tensor $L$ of type $(1,3)$ over $V$ is a bilinear mapping $L: V \times V \rightarrow \operatorname{Hom}(V, V),(x, y) \mapsto L(x, y)$. $L$ is called a curvature tensor if it has the following properties for all $x, y, z, w$ $\in V:$

(i ) $L(x, y)=-L(y, x)$;

(ii) $L(x, y)$ is a skew symmetric endomorphism of $V$, i. e.

where

$$
L(x, y, z, w)+L(x, y, w, z)=0,
$$

$$
L(x, y, z, w)=g(L(x, y) z, w)
$$

(iii) $\underset{x, y, z}{\subseteq} L(x, y) z=0$ where $\subseteq$ denotes the cyclic sum (this is the first Bianchi identity).

This means also that $L$ is a symmetruc double form of type $(2,2)$ which satisfies the first Bianchi identity [8], [13].

The Ricct tensor $L_{R}$ of type $(0,2)$ associated with $L$ is the symmetric bilinear function on $V \times V$ defined by $L_{R}(x, y)=\operatorname{trace}(z \in V \mapsto L(z, x) y \in V)$. The Ricci tensor $Q=Q_{R}(L)$ of type $(1,1)$ is given by $L_{R}(x, y)=g(Q x, y)$ and the trace of $Q$ 
is called the scalar curvature $l=l(L)$ of $L$.

Let $P$ be a 2-plane of $V$ with orthonormal basis $\{x, y\}$. Then $k_{L}(P)=$ $g(L(x, y) y, x)$ is independent of the chosen orthonormal basis and is called the $L$-sectronal curvature of $P$. If the function $k_{L}: P \rightarrow k_{L}(P)$ is constant, we say that $L$ has constant sectional curvature.

The curvature tensors $L$ over $V$ form a subspace of the vector space of $(1,3)$-tensors over $V$, and we denote this subspace by $\mathcal{K}(V)$. It has a natural inner product $\langle$,$\rangle induced from that on V$ :

$$
\langle L, \widetilde{L}\rangle=\sum_{\imath, j, k=1}^{n} g\left(L\left(e_{\imath}, e_{\jmath}\right) e_{k}, \widetilde{L}\left(e_{\imath}, e_{j}\right) e_{k}\right),
$$

$\left\{e_{i}\right\}$ being an arbitrary orthonormal basis of $V$.

Next, let $V$ be a $(2 n+1)$-dimensional real vector space with a cocomplex structure $(\varphi, \xi, \eta)$ and a co-Hermitian scalar product $g$, i. e. $\varphi$ is a linear operator on $V, \xi \neq 0 \in V$ and $\eta \in V^{*}$ (the dual space of $V$ ) such that

$$
\begin{gathered}
\varphi^{2}=-I+\eta \otimes \xi, \\
\eta(\xi)=1, \\
\forall x, y \in V: g(\varphi x, \varphi y)=g(x, y)-\eta(x) \eta(y) .
\end{gathered}
$$

$I$ is the identity transformation on $V$. From this we deduce at once

$$
\varphi(\xi)=0, \eta \bullet \varphi=0, \operatorname{Im} \varphi=\operatorname{Ker} \eta=\{\xi\}^{\perp}, g(\xi, \xi)=1,
$$

and $\eta$ is the dual vector of $\xi$. Further, every co-Hermitian vector space has a special type of orthonormal basis, called a $\varphi$-basis, consisting of $\left(e_{\imath}, \varphi e_{\imath}, \xi\right.$; $\imath=1, \cdots, n)$. A 2-plane $P$ of $V$ containing $\xi$ is called a $\xi$-plane while a 2-plane $P \subset\{\xi\}^{\perp}$ is called a $\varphi$-plane. A $\varphi$-plane for which $\varphi(P)=P$ is a holomorphrc $\varphi$-plane. Such plane has an orthonormal basis of type $(x, \varphi x)$ with $g(\xi, x)=0$.

Now let $L$ be a curvature tensor over the co-Hermitian vector space $V$. The restriction of $k_{L}$ to a $\xi$-plane $P$ is called the $\xi$-sectional curvature of $P$ and the restriction to a holomorphic $\varphi$-plane $P$ is called the $\varphi$-holomorphic sectional curvature of $P$. This will be denoted by $\varphi_{L}$.

Based on theorem 2.3 and definition 2.4 we consider now a special class of curvature tensors over $V$.

Definition 3.1. A C-curvature tensor $L$ over a co-Hermitian vector space $V$ is a curvature tensor such that

$$
L(x, y, z, w)=L(x, y, \varphi z, \varphi w)+\alpha\{g(\wedge(x, y) z, w)-g(\wedge(x, y) \varphi z, \varphi w)\}
$$

for an $\alpha \in \boldsymbol{R}$ and for all $x, y, z, w \in V$. Here $\wedge$ is the curvature tensor defined by $\wedge(x, y) z=(x \wedge y) z=g(y, z) x-g(x, z) y$. The number $\alpha$ is called the type of $L$ and a $C$-curvature tensor of type $\alpha$ is called a $C(\alpha)$-curvature tensor.

A C(1)-curvature tensor is a Sasakian curvature tensor; a $C(0)$-curvature 
tensor is a co-Kähler or CK-curvature tensor and a $C(-1)$-curvature tensor is a Kenmotsu curvature tensor.

The set of all $C(\alpha)$-curvature tensors over $V$ will be denoted by $\mathcal{K}_{C(\alpha)}(V)$ and the union of all $\mathcal{K}_{C(\alpha)}(V), \alpha \in \boldsymbol{R}$ (thus the set of all $C$-curvature tensors) is a subspace of $\mathcal{K}(V)$. For this space we use the notation $\mathcal{K}_{C}(V)$. We note that $\mathcal{K}_{C(0)}(V)=C K(V)$ is a subspace of $\mathcal{K}_{C}(V)$.

As one can see from the definition, there is a strong relation between the notion of type of a $C$-curvature tensor and the notion of type of a $K_{3}$-curvature tensor on a Hermitian vector space [24], [25], [27], [29].

To precise the analogy of $C K$-tensors with $K_{1}$-tensors (or Kähler curvature tensors on a Hermitian vector space [16]) we consider the notion of a cocomplex linear map.

Definition 3.2. Let $V(\varphi, \xi, \eta)$ and $V^{\prime}\left(\varphi^{\prime}, \xi^{\prime}, \eta^{\prime}\right)$ be cocomplex vector spaces over $\boldsymbol{R}$. A linear map $\varphi: V \rightarrow V^{\prime}$ is cocomplex if

(i) $\varphi^{\prime} \circ f=f \circ \varphi$;

(ii) $\exists k \in \boldsymbol{R}^{+}$such that $f \xi=k \xi^{\prime}$;

(iii) $\exists l \in \boldsymbol{R}^{+}$such that $f^{*} \eta^{\prime}=l \eta$.

It follows easily that for a cocomplex map $k=l$ and that an orthogonal map $f: V \rightarrow V^{\prime}$, where $V$ and $V^{\prime}$ are co-Hermitian, is cocomplex linear if and only if $\varphi^{\prime} \circ f=f \circ \varphi$ and $f \xi=\xi^{\prime}$.

THEOREM 3.3. $L \in \mathcal{K}(V)$ is a CK-tensor over $V$ if and only if $L(x, y)$ is cocomplex linear for all $x, y \in V$.

Proof. Let $L(x, y)$ be cocomplex linear. Then $L(x, y) \xi=k \xi$ and so $g(L(x, y) \xi, \xi)=L(x, y, \xi, \xi)=0=k g(\xi, \xi)=k$. This means that $L(x, y, z, \xi)=0$ for $x, y, z \in V$. Further we have

$$
g(L(x, y) \varphi z, \varphi w)=g(\varphi L(x, y) z, \varphi w)=g(L(x, y) z, w)-\eta(L(x, y) z) \eta(w)
$$

and so $L(x, y, \varphi z, \varphi w)=L(x, y, z, w)$.

Conversely, let $L$ be a $C K$-tensor. Then we have for all $x, y, z \in V$ that $L(x, y, \xi, w)=L(x, y, \varphi \xi, \varphi w)=0$ and thus $L(x, y) \xi=0$. Further $\eta(L(x, y) z)=$ $L(x, y, z, \xi)=0$ and

$$
\begin{aligned}
g(\varphi(L(x, y) z), \varphi w) & =g(L(x, y) z, w)-\eta(L(x, y) z) \eta(w) \\
& =g(L(x, y) z, w)=g(L(x, y) \varphi z, \varphi w) .
\end{aligned}
$$

Since this holds for all $\varphi w$ and also for $\xi$, we get $\varphi \circ L(x, y)=L(x, y) \circ \varphi$.

The following theorem is an immediate consequence of the definition of a $C(\alpha)$-curvature tensor. 
THEOREM 3.4. If $L \in \mathcal{K}_{C(\alpha)}(V)$, then

(i) $\forall x \in V, \forall y, z \in\{\xi\}^{\perp}: L(y, z, x, \xi)=0$;

(ii) $\forall x, y \in V: L(x, \xi, y, \xi)=-\alpha g(\varphi x, \varphi y)$.

If $L \in C K(V)$, then

(iii) $\forall x \in V: L(x, \xi)=0$;

(iv) $\forall x, y, z, w \in\{\xi\}^{\perp}: L(x, y, z, w)=L(x, y, \varphi z, \varphi w)$.

For each $L \in \mathcal{K}(V)$ we construct an $\alpha$-associated curvature tensor $P(L, \alpha)$ by

$$
P(L, \alpha)(x, y)=L(x, y)-\alpha \wedge(x, y)
$$

and it follows that $L \in \mathcal{K}_{C(\alpha)}(V)$ if and only if $P(L, \alpha) \in C K(V)$. So the spaces $\mathcal{K}_{C(\alpha)}(V)$ are affine subspaces of $\mathcal{K}(V)$ for each $\alpha$. The $C(\alpha)$-curvature tensor $L$ has constant $\varphi$-holomorphic sectional curvature $c$ if and only if $P(L, \alpha)$ has constant $\varphi$-holomorphic sectional curvature $c-\alpha$.

Concerning the sectional curvatures of $L \in \mathcal{K}_{C(\alpha)}(V)$, we see at once that the $\xi$-sectional curvature is always constant and equal to $\alpha$. For the case of constant $\varphi$-holomorphic sectional curvature we have an explicit expression for $L$.

THEOERM 3.5. $L \in \mathcal{K}_{C(\alpha)}(V)$ has constant $\varphi$-holomorphic sectional curvature $c$ if and only if for all $x, y, z \in V$

$$
\begin{aligned}
L(x, y) z= & \frac{c+3 \alpha}{4} \wedge(x, y) z+\frac{c-\alpha}{4}\{\wedge(\varphi x, \varphi y) z+2 g(x, \varphi y) \varphi z \\
& +\eta(x) y \eta(z)-\eta(y) x \eta(z)+\eta(y) \xi g(x, z)-\eta(x) \xi g(y, z)\} .
\end{aligned}
$$

Proof. Let $P \in C K(V)$ with constant $\varphi$-holomorphic sectional curvature $c-\alpha$. By a well known linearization technique we get for all $x, y, z, w \in\{\xi\}^{\perp}$ :

$$
\begin{aligned}
P(x, y, z, w)=\frac{c-\alpha}{4} & \{g(x, w) g(y, z)-g(y, w) g(x, z)-g(x, \varphi w) g(z, \varphi y) \\
& +g(y, \varphi w) g(z, \varphi x)+2 g(\varphi x, y) g(z, \varphi w)\} .
\end{aligned}
$$

Now, let $x \in V$. Then $x=-\varphi^{2} x+\eta(x) \xi$. Hence for all $x, y, z, w \in V$

$$
P(x, y, z, w)=P\left(\varphi^{2} x, \varphi^{2} y, \varphi^{2} z, \varphi^{2} w\right) .
$$

Since $\varphi^{2} x \in\{\xi\}^{\perp}$ and $\left(\varphi^{3}+\varphi\right) x=0$ we obtain easily

$$
\begin{aligned}
P(x, y) z=\frac{c-\alpha}{4} & \{\wedge(x, y) z+\wedge(\varphi x, \varphi y) z+2 g(x, \varphi y) \varphi z \\
& +\eta(x) y \eta(z)-\eta(y) x \eta(z)+\eta(y) \xi g(x, z)-\eta(x) \xi g(y, z)\} .
\end{aligned}
$$

The result follows now from $L(x, y) z=P(x, y) z+\alpha \wedge(x, y) z$.

This result is well known for $C(1)$-curvature tensors [4], $C(0)$-curvature tensors [14] and $C(-1)$-curvature tensors [12]. There is a formal analogy with 
the expression for the curvature tensor of a generalized complex space form [26].

Just as for Kähler curvature tensors, the behaviour of the Ricci tensor plays an important role in the decomposition theory (the tensor is complex linear for $K$-curvature tensors). We have

THEOREM 3.6. Let $L \in \mathcal{K}_{C}(V)$. Then $Q_{R}(L)$ is cocomplex linear.

Proof. Let $P$ denote the associated curvature tensor of $L$. Then

$$
Q_{R}(P)=Q_{R}(L)-2 n \alpha I .
$$

Since $P \in C K(V)$ we have $g\left(Q_{R}(P) x, \xi\right)=\sum_{\imath=1}^{2 n+1} P\left(x, e_{\imath}, e_{\imath}, \xi\right)=0$ where $\left\{e_{i}\right\}$ is an orthonormal basis of $V$. Hence $Q_{R}(P) \xi=0$ and so $Q_{R}(L) \xi=2 n \alpha \xi$.

Next we have $g\left(Q_{R}(L) x, \xi\right)=g\left(x, Q_{R}(L) \xi\right)=0$ for $\eta \in\{\xi\}^{\perp}$ and so $\eta\left(Q_{R}(L) x\right)$ $=0$. Further $\eta\left(Q_{R}(L) \xi\right)=2 n \alpha$; so $\eta \circ Q_{R}(L)=2 n \alpha \eta$.

Finally we prove $\varphi^{\circ} Q_{R}(L)=Q_{R}(L) \circ \varphi$. Let $\left\{e_{\imath}, \varphi e_{\imath}, \xi, \imath=1, \cdots, n\right\}$ be a $\varphi$-basis for $V$. Then for $x, y \in\{\xi\}^{\perp}$

$$
g\left(Q_{R}(L) \varphi x, y\right)=\sum_{\imath=1}^{2 n} L\left(\varphi x, e_{\imath}, e_{\imath}, y\right)+L(\varphi x, \xi, \xi, y) .
$$

Now

$$
\sum_{\imath=1}^{2 n} L\left(\varphi x, e_{\imath}, e_{\imath}, y\right)=\sum_{\imath=1}^{2 n} L\left(\varphi^{2} x, \varphi e_{\imath}, \varphi e_{\imath}, \varphi y\right)=-\sum_{\imath=1}^{2 n} L\left(x, e_{\imath}, e_{\imath}, \varphi y\right)
$$

and

Hence

$$
L(\varphi x, \xi, \xi, y)=\alpha g\left(\varphi^{2} x, \varphi y\right)=-\alpha g(x, \varphi y)=-L(x, \xi, \xi, \varphi y) .
$$

$$
g\left(Q_{R}(L) \varphi x, y\right)=-g\left(Q_{R}(L) x, \varphi y\right)=g\left(\varphi Q_{R}(L) x, y\right) .
$$

Since $g\left(Q_{R}(L) \varphi x, \xi\right)=0=g\left(\varphi Q_{R}(L) x, \xi\right)$ we obtain for $x \in\{\xi\}^{\perp}$

Finally

$$
\left(Q_{R}(L) \circ \varphi\right) x=\left(\varphi \circ Q_{R}(L)\right) x .
$$

$$
\left(\varphi \circ Q_{R}(L)\right) \xi=\varphi(2 n \alpha \xi)=0=\left(Q_{R}(L) \circ \varphi\right) \xi,
$$

which proves the required result.

To finisch this section we give some examples of $C$-curvature tensors we need later on. We frequently use the curvature tensor $\mathscr{H}$ over $V$, defined by

$$
\mathscr{H}(x, y) z=\eta(x) y \eta(z)-\eta(y) x \eta(z)+\eta(y) \xi g(x, z)-\eta(x) \xi g(y, z) .
$$

Further $\operatorname{Hom}^{\cos }(V)$ denotes the space of all symmetric cocomplex linear endomorphisms of the co-Hermitian vector space $(V, \varphi, \xi, \eta, g)$ with dimension $2 n+1$.

Example 3.7. Let $a, \alpha \in \boldsymbol{R}$ and $A, B \in \operatorname{Hom}^{\cos }(V)$. Then $C_{A, B, a, \alpha}$ with 


$$
\begin{aligned}
C_{A, B, a, \alpha}(x, y)= & \wedge(A x, A y)+\wedge(B x, A y)+\wedge(\varphi A x, \varphi B y)+\wedge(B x, \varphi A y) \\
& +2 g(A x, \varphi y) \varphi B-2 g(\varphi x, B y) \varphi A+\mathcal{H}(A x, B y)+\mathscr{H}(B x, A y) \\
& +a \wedge(x, y)+(a-\alpha)\{\wedge(\varphi x, \varphi y)+\mathscr{H}(x, y)+2 g(x, \varphi y) \varphi\}
\end{aligned}
$$

is a $C(\alpha)$-curvature tensor.

If $A \xi=k \xi$ and $B \xi=l \xi$, we obtain

$$
\begin{aligned}
& Q_{R}\left(C_{A, B, a, \alpha}\right)=\operatorname{tr} B-l) A+(\operatorname{tr} A-k) B+2(A B+B A) \\
&+2\{a(n+1)-\alpha\} I-\{2 k l+k \operatorname{tr} B+l \operatorname{tr} A+2(n+1)(a-\alpha)\} \eta \otimes \xi, \\
& l\left(C_{A, B, a, \alpha}\right)=2 \operatorname{tr} A \operatorname{tr} B-2 l \operatorname{tr} A-2 k \operatorname{tr} B+2 \operatorname{tr} A B+2 \operatorname{tr} B A \\
&-2 k l+2 n\{2 a(n+1)-\alpha\} .
\end{aligned}
$$

Some special cases have special importance later on. Therefore we put

$$
C_{A, B, \alpha}=C_{A, B, \frac{3 \alpha}{4}, \alpha}, \quad C_{A, B}=C_{A, B, 0} .
$$

EXAMPLE 3.8. For $C=C_{\frac{c}{8} I, I, \alpha}(c \in \boldsymbol{R})$ we have $k=\frac{c}{8}, l=1$ and

$$
\begin{aligned}
& C(x, y)=\frac{c+3 \alpha}{4} \wedge(x, y)+\frac{c-\alpha}{4}\{\wedge(\varphi x, \varphi y)+\mathscr{H}(x, y)+2 g(x, \varphi y) \varphi\}, \\
& Q_{R}(C)=\frac{n(c+3 \alpha)+c-\alpha}{2} I-\frac{(n+1)(c-\alpha)}{2} \eta \otimes \xi, \\
& l(C)=\frac{1}{2}\{n(2 n+1)(c+3 \alpha)+n(c-\alpha)\} .
\end{aligned}
$$

$C$ has constant $\varphi$-holomorphic sectional curvature $c$. By theorem 3.5 we know that conversely, any $C(\alpha)$-curvature tensor with constant $\varphi$-holomorphic sectional curvature is of this form $C$.

\section{The decomposition theorem.}

Let $(V, g, \varphi, \xi, \eta)$ be a co-Hermitian vector space of dimension $2 n+1$ and $\mathcal{K}_{C}(V)$ the subspace of $\mathcal{K}(V)$ formed by the $C$-curvature tensors. The natural induced inner product on $\mathcal{K}_{C}(V)$ will also be denoted by $g$. Just as for the Weyl tensor [17], [20] and for the Bochner tensor [16], [21], we determine a decomposition of $\mathcal{K}_{C}(V)$ into a direct sum of orthogonal subspaces. The considered subspaces are in some sense inspired by the decomposition given in [24], [27] for $K_{3}$-curvature tensors. In section 5 we shall show that the components are irreducible for a natural induced representation of $\mathcal{U}(n) \times 1$ on $\mathcal{K}_{C}(V)$.

LEMmA 4.1. Let $\mathcal{C}_{1}(V)=\left\{L \in \mathcal{K}_{C}(V) \mid \varphi_{L}=0\right\}$. Then for $L \in \mathcal{C}_{1}(V)$ we have 


$$
L(x, y)=\frac{\alpha}{4}\{3 \wedge(x, y)-\wedge(\varphi x, \varphi y)-\mathscr{H}(x, y)-2 g(x, \varphi y) \varphi\},
$$

where $\alpha \in \boldsymbol{R}$.

Proof. This is a special case of theorem 3.5 with $c=0 . \quad \mathcal{C}_{1}(V)$ is thus a 1-dimensional subspace of $\mathcal{K}_{C}(V)$.

LEMMA 4.2. Let $\mathcal{C}_{1}^{\perp}(V)$ be the orthogonal complement of $\mathcal{C}_{1}(V)$ in $\mathcal{K}_{C}(V)$. Then $\mathcal{C}_{1}^{\perp}(V)=C K(V)$.

Proof. Let $L \in \mathcal{C}_{1}(V)$ with type $\alpha$ and let $\widetilde{L}$ be an arbitrary element of $\mathcal{K}_{C}(V)$. We take an orthonormal basis $\left\{e_{a}, \varphi e_{a}, \xi ; a=1, \cdots, n\right\}$ and put $e_{2 n+1}=\xi$, $e_{a *}=\varphi e_{a}, a^{*}=n+a$. We consider now

$$
g(L, \widetilde{L})=\sum_{A, B, C, D=1}^{2 n+1} L_{A B C D} \widetilde{L}_{A B C D},
$$

where $L_{A B C D}=L\left(e_{A}, e_{B}, e_{C}, e_{D}\right)$. When $A=2 n+1$ we write $L_{A B C D}$ as $L_{\xi B C D}$.

First we have by lemma 4.1

$$
\begin{aligned}
L_{\imath j k l} & =\frac{\alpha}{4}\left\{3 \delta_{k j} \delta_{i l}-3 \delta_{k i} \delta_{j l}-\delta_{k j *} \delta_{l i *}+\delta_{k i *} \delta_{\jmath l *}+2 \delta_{i j *} \delta_{k l *}\right\}, \\
L_{i \xi \xi k} & =\alpha \delta_{i k} .
\end{aligned}
$$

Hence

$$
\begin{aligned}
g(L, \widetilde{L}) & =\sum_{\imath, \jmath, k, l=1}^{2 n} L_{\imath j k l} \widetilde{L}_{\imath j k l}+4 \sum_{\imath, k=1}^{2 n} L_{i \xi k \xi} \widetilde{L}_{i \xi k \xi} \\
& =\frac{3 \alpha}{2} \sum_{\imath, \jmath=1}^{2 n} \widetilde{L}_{\imath j j i}-\frac{\alpha}{2} \sum_{\imath, \jmath=1}^{2 n}\left(\widetilde{L}_{\imath \jmath \jmath * i *}+\widetilde{L}_{i * \imath \jmath j *}\right) .
\end{aligned}
$$

Using the first Bianchi identity and the fact that $\widetilde{L}$ is a $C\left(\alpha^{\prime}\right)$-curvature tensor, we get

$$
g(L, \tilde{L})=2 n(3 n+1) \alpha \alpha^{\prime} .
$$

Hence, $\tilde{L} \in \mathcal{C}_{1}^{\perp}(V)$ if and only if $\alpha^{\prime}=0$.

LEMMA 4.3. Let $\mathcal{C}_{2}(V)=\left\{L \in C K(V)\right.$ with $\varphi_{L}$ constant $\}$. Then for $L \in \mathcal{C}_{2}(V)$ we have

$$
L(x, y)=\frac{c}{4}\{\wedge(x, y)+\wedge(\varphi x, \varphi y)+\mathscr{H}(x, y)+2 g(x, \varphi y) \varphi\},
$$

where $c \in \boldsymbol{R}$.

Proof. Apply theorem 3.5 with $\alpha=0$. So $\mathcal{C}_{2}(V)$ is a 1-dimensional subspace of $C K(V)$. 
Now let $\tilde{C}(V)$ be the orthogonal complement of $\mathcal{C}_{1}(V) \oplus \mathcal{C}_{2}(V)$ in $\mathcal{K}_{C}(V)$. Then we have

LEMMA 4.4. $\tilde{C}(V)=\{L \in C K(V)$ with $l(L)=0\}$.

Proof. Let $L \in \mathcal{C}_{1}(V) \oplus \mathcal{C}_{2}(V)$ and $\tilde{L} \in \mathcal{C}(V)$. Lemma 4.2 implies that $\tilde{L} \in C K(V)$. From theorem 3.5 we have

$$
L(x, y)=\frac{c+3 \alpha}{4} \wedge(x, y)+\frac{c-\alpha}{4}\{\wedge(\varphi x, \varphi y)+\mathscr{H}(x, y)+2 g(x, \varphi y) \varphi\} .
$$

Hence

$$
\begin{aligned}
g(L, \widetilde{L}) & =\sum_{\imath, j, k, l=1}^{2 n} L_{\imath j k l} \widetilde{L}_{\imath j k l}+4 \sum_{\imath, k=1}^{2 n} L_{i \xi k \xi} \widetilde{L}_{\imath \xi k \xi} \\
& =\frac{c+3 \alpha}{2} \sum_{\imath, j=1}^{2 n} \widetilde{L}_{\imath \jmath j i}+\frac{3(c-\alpha)}{2} \sum_{i, j=1}^{2 n} \widetilde{L}_{\imath \jmath j i} .
\end{aligned}
$$

Further, since $\tilde{L}_{i \xi i}=0$ we have

$$
g(L, \widetilde{L})=2 c l(\widetilde{L}),
$$

which proves the required result.

LEMMA 4.5. Let $\mathcal{C}_{B}(V)=\left\{L \in C K(V)\right.$ with $\left.Q_{R}(L)=0\right\}$. Then $C_{B}(V)$ is a subspace of $\tilde{C}(V)$.

Proof. Trivial.

So we get

THEOREM 4.6. We have

$$
\mathcal{K}_{C}(V)=\mathcal{C}_{1}(V) \oplus \mathcal{C}_{2}(V) \oplus \mathcal{C}_{B}(V) \oplus \mathcal{C}_{3}(V) \quad \text { (orthogonal) }
$$

where $\mathcal{C}_{3}(V)$ is the orthogonal complement of $\mathcal{C}_{B}(V)$ in $\tilde{C}(V)$.

To find the dimensions of the subspaces $\mathcal{C}_{3}(V)$ and $\mathcal{C}_{B}(V)$ we use essentially a result proved in [21].

THEOREM 4.7. If $(V, \varphi, \xi, \eta, g)$ is a $(2 n+1)$-dimensional co-Hermitzan vector space, then $\operatorname{dim} \mathcal{K}_{C}(V)=\frac{n^{2}(n+1)^{2}}{4}+1, \quad \operatorname{dim} \mathcal{C}_{3}(V)=n^{2}-1 \quad$ and $\quad \operatorname{dim} \mathcal{C}_{B}(V)=$ $\frac{1}{4} n^{2}(n-1)(n+3)$.

Proof. Let $W=\operatorname{Ker} \eta$. Then $W$ is a Hermitian vector space with $(g|W, \varphi| W)$ as Hermitian structure. We consider $\mathcal{K}_{1}(W)$, the space of curvature tensors $L$ over $W$ satisfying the Kähler identity $L(x, y, z, w)=L\left(x, y,\left.\varphi\right|_{W} z,\left.\varphi\right|_{W} w\right)$. It is 
clear that $\mathcal{K}_{1}(W)$ is isomorphic with $C K(V)$. Indeed, the restriction $L_{1}$ of $L \in C K(V)$ to $W$ belongs to $\mathcal{K}_{1}(V)$ and conversely any $L_{1} \in \mathcal{K}_{1}(W)$ can be extended uniquely to an element $L$ of $C K(V)$ (see theorems 3.3 and 3.4). Further, $Q_{R}\left(L_{1}\right)$ $=0$ if and only if $Q_{R}(L)=0$ and the holomorphic $\varphi$-sectional curvature of $L$ is the holomorphic sectional curvature of $L_{1}$. This means that $\mathcal{C}_{2}(V), \mathcal{C}_{B}(V), \mathcal{C}_{3}(V)$ are isomorphic with the subspaces in the decomposition of $\mathcal{K}_{1}(W)$ given in [16], [21]. The dimensions of these subspaces and of $\mathcal{K}_{1}(W)$ are given in [21]. This gives the required result.

Now let $L \in \mathcal{K}_{C(\alpha)}(V)$. Then we have

$$
L=\sum_{i=1}^{3} L_{i}+L_{B}
$$

where $L_{\imath} \in C_{\imath}(V)$ and $L_{B} \in \mathcal{C}_{B}(V)$. We determine the explicit expressions for these projections.

First, $L_{1}$ has to be a $C(\alpha)$-tensor and the expression is given by lemma 4.1 . To know $L_{2}$ we use lemma 4.3 and determine $c$. Since $l(L)=l\left(L_{1}\right)+l\left(L_{2}\right)$ (using lemma 4.4) we get from example 3.8

$$
c=\frac{l(L)-\alpha n(3 n+1)}{n(n+1)}
$$

and hence

$$
L_{2}(x, y)=\frac{l(L)-\alpha n(3 n+1)}{4 n(n+1)}\{\wedge(x, y)+\wedge(\varphi x, \varphi y)+\mathcal{H}(x, y)+2 g(x, \varphi y) \varphi\} .
$$

To determine $L_{3}$ we prove

LEMmA 4.8. For all $a, b \in \boldsymbol{R}$ and all $A \in \mathrm{Hom}^{\cos }(V)$, the CK-curvature tensors $L$ defined by

belong to $\mathcal{C}_{B}^{\perp}(V)$.

$$
L=a C_{A, I}+b C_{I, I}
$$

Proof. Let $\tilde{L} \in \mathcal{C}_{B}(V)$. Then $\tilde{L} \in C K(V)$ and $\tilde{L}_{R}=0$. Further, since $A \in$ $\mathrm{Hom}^{\cos }(V), \varphi x$ is an eigenvector of $A$ if $x$ is an eigenvector and both have the same eigenvalue. Hence we may choose a $\varphi$-basis of eigenvectors $\left\{e_{a}, \varphi e_{a}, \xi\right.$; $a=1, \cdots, n\}$ for $V$. We have

$$
g(L, \widetilde{L})=\sum_{l, \jmath, k, l=1}^{2 n} L_{\imath \jmath k l} \widetilde{L}_{\imath \jmath k l}
$$

and using example 3.7 we obtain

$$
g(L, \tilde{L})=8 \sum_{\imath, j=1}^{2 n}\left\{a\left(\lambda_{2}+\lambda_{j}\right)+2 b\right\} \widetilde{L}_{\imath \jmath \jmath \imath}
$$

where $\lambda_{\imath}$ is the eigenvalue of $e_{\imath}$. So 


$$
g(L, \tilde{L})=8 a \sum_{\imath=1}^{2 n} \lambda_{i}\left(\widetilde{L}_{R}\right)_{\imath \imath}+8 a \sum_{\jmath=1}^{2 n} \lambda_{\jmath}\left(\widetilde{L}_{R}\right)_{\jmath \jmath}+16 b \sum_{\imath=1}^{2 n}\left(\widetilde{L}_{R}\right)_{i i} .
$$

Since $\widetilde{L}_{R}=0$ we get $g(L, \widetilde{L})=0$.

Now we put $L_{3}=a C_{A, I}+b C_{I, I}$ and determine $a, b$ and $A$ so that $L_{R}=$ $L_{1 R}+L_{2 R}+L_{3 R}$. This implies immediately that $A=Q_{R}(L)$, which is indeed cocomplex linear, and

$$
a=\frac{1}{2(n+2)}, \quad b=\frac{2 \alpha n-l(L)}{4 n(n+2)}
$$

We put

$$
k=\frac{l(L)+2 \alpha n}{2(n+1)}
$$

Then we have proved

Theorem 4.9. Let $L \in \mathcal{K}_{C(r)}(V)$. Then $L=\sum_{i=1}^{3} L_{\imath}+L_{B}$ with $L_{i} \in \mathcal{C}_{i}(V)$ and $L_{B} \in \mathcal{C}_{B}(V)$. Further

$$
\begin{aligned}
L_{1}(x, y)= & \frac{\alpha}{4}\{3 \wedge(x, y)-\wedge(\varphi x, \varphi y)-\mathscr{H}(x, y)-2 g(x, \varphi y) \varphi\}, \\
L_{2}(x, y)= & \frac{2 k-3 \alpha n}{8 n} C_{I I}(x, y), \\
L_{3}(x, y)= & \frac{1}{2(n+1)} C_{Q_{R}(L), I}(x, y)+\frac{2 \alpha n-k(n+1)}{2 n(n+2)} C_{I, I}(x, y), \\
L_{B}(x, y)= & L(x, y)-\frac{1}{2(n+2)} C_{Q_{R}(L), I}(x, y)+\frac{k}{4(n+2)} C_{I, I}(x, y) \\
& +\frac{\alpha}{n+2}\{-2 \wedge(x, y)+n \wedge(\varphi x, \varphi y)+\mathscr{H}(x, y)+2 g(x, \varphi y) \varphi\} .
\end{aligned}
$$

Definition 4.10. Let $L \subseteq \mathcal{K}_{C}(V)$. Then $L_{B}$ is called the $C$-Bochner tensor associated with $L$. $L$ is called C-Bochner flat if $L_{B}=0$.

This allows us to introduce now in a natural way the notion of C-Bochner curvature tensor on an almost $C(\alpha)$-manifold. Indeed, in every tangent space the Riemann curvature tensor $R$ defines a $C(\alpha)$-curvature tensor. By taking the corresponding $R_{B}$-component at each point, we get the $C$-Bochner curvature tensor field $B$ on the almost $C(\alpha)$-manifold $M$. If $B=0, M$ is called a $C$-Bochner flat manifold.

For Sasakian manifolds our definition coincides with the tensor introduced, using the Bootby-Wang fibration, in [15] (see also [32]). 


\section{The irreducibility of the decomposition.}

The decomposition constructed in section 4 can be characterized completely by showing that the components are the irreducible components of the induced natural representation of $U(n) \times 1$ in the space $\mathcal{K}_{C}(V)$. The decompositions given in [20], [17], [16], [21] have the same irreducibility property if one considers the structure group $\mathcal{O}(n)$ resp. $U(n)$. This may be proved using Weyl's invariants theorem and the theory of quadratic invariants of the curvature tensor (see [1], [10], [11], [31]).

Let $V$ be a $(2 n+1)$-dimensional co-Hermitian vector space and let $\rho$ be the standard representation of $U(n) \times 1$ in $V$. Thus

where

$$
\rho: \mathcal{U}(n) \times 1 \rightarrow G L(V) ; X \mapsto \rho(X),
$$

$$
\rho(X): V \rightarrow V ; v \mapsto\left(\begin{array}{rrr}
A & B & 0 \\
-B & A & 0 \\
0 & 0 & 1
\end{array}\right) v .
$$

The representation $\rho$ induces a representation $\tilde{\rho}$ of $U(n) \times 1$ in the space $\mathcal{K}(V)$ by

where

$$
\tilde{\rho}: \mathcal{U}(n) \times 1 \rightarrow G L(\mathcal{K}(V)), \quad g \mapsto \tilde{\rho}(g),
$$

$$
\tilde{\rho}(g)(L)(x, y, z, w)=L\left(\rho\left(g^{-1}\right) x, \rho\left(g^{-1}\right) y, \rho\left(g^{-1}\right) z, \rho\left(g^{-1}\right) w\right) .
$$

$U(n) \times 1$ is the group of all orthogonal cocomplex linear transformations. So $g\left(X^{-1} x, X^{-1} z\right)=g(x, z)$ and $\varphi^{\circ} X^{-1}=X^{-1} \circ \varphi$ for all $X \in \mathcal{U}(n) \times 1$. This implies

THEOREM 5.1. $\mathcal{K}_{C}(V)$ is an invariant subspace of $\mathcal{K}(V)$ for $\tilde{\rho}$.

Further we have for $\tilde{\rho}$.

THEOREM 5.2. $\quad \mathcal{C}_{i}(V)(\imath=1,2,3)$ and $\mathcal{C}_{B}(V)$ are invarnant subspaces of $\mathcal{K}_{C}(V)$

Proof. This is a straightforward verification using the fact that elements of $U(n) \times 1$ are cocomplex linear and orthogonal, together with the defining properties of the subspaces.

Now we prove that there are precisely four irreducible components for the representation $\tilde{\rho}$. Therefore we shall use the notion of the direct sum of two representations.

LEMMA 5.3. Let $\rho: G \rightarrow G L(V)$ be a representation with a irreducible components and $\rho^{\prime}: G^{\prime} \rightarrow G L\left(V^{\prime}\right)$ a representation with $b$ irreducible components. $G, G^{\prime}$ are groups and $V, V^{\prime}$ subspaces of a real vector space $W$. Then 
defined by

$$
\rho+\rho^{\prime}: G \times G^{\prime} \rightarrow G L\left(V \oplus V^{\prime}\right)
$$

$$
\left(\rho+\rho^{\prime}\right)\left(g, g^{\prime}\right)\left(v+v^{\prime}\right)=\rho(g) v+\rho^{\prime}\left(g^{\prime}\right) v^{\prime}
$$

is a representation with $a+b$ irreducible components.

THEOREM 5.4. The representation $\tilde{\rho}$ of $U(n) \times 1$ in $\mathcal{K}_{C}(V)$ is a direct sum of two representations.

Proof. As in the proof of theorem 4.7 we consider $\mathcal{K}_{1}(W)$ with $W=\operatorname{Ker} \eta$. Let $\sigma: \mathcal{V}(n) \rightarrow G L(W)$ be the standard representation of $U(n)$ in $W$ and $\tilde{\sigma}$ the induced representation of $U(n)$ in $\mathcal{K}_{1}(W)$.

Next, let 1: $\{e\} \rightarrow G L\left(\mathcal{C}_{1}(V)\right)$ be the trivial representation. Using the isomorphism between $C K(V)$ and $\mathcal{K}_{1}(W)$ (see theorem 4.7) and identifying

$$
\left(\begin{array}{rrr}
A & B & 0 \\
-B & A & 0 \\
0 & 0 & 1
\end{array}\right) \text { with }\left(\left(\begin{array}{rr}
A & B \\
-B & A
\end{array}\right), 1\right)
$$

together with the property $\tilde{\rho}(X) L=L$ for $L \in \mathcal{C}_{1}(V)$, we obtain that the direct sum $\tilde{\sigma}+1$ is precisely $\tilde{\rho}$.

Since $\tilde{\sigma}$ has three irreducible components if $\operatorname{dim} W>2$ we obtain by lemma 5.3 that $\tilde{\rho}$ has four irreducible components if $\operatorname{dim} V \geqq 5$. Hence we have

THEOREM 5.5. The decomposition of theorem 4.6 is a decomposition in irreducible components if $\operatorname{dim} V \geqq 5$.

In the case of $\operatorname{dim} V=3$ we have only two irreducible components for $\tilde{\rho}$ (then $\operatorname{dim} W=2)$ and $\mathcal{K}_{C}(V)=\mathcal{C}_{1}(V) \oplus \mathcal{C}_{2}(V)$, according with the result of theorem 4.7. This implies that the $C$-Bochner tensor vanishes for $C(\alpha)$-curvature tensors over $V$ if $\operatorname{dim} V=3$.

\section{Applications.}

We shall give now some natural applications of the decomposition. They will show the analogy of the $C$-Bochner tensor with the classical Weyl and Bochner tensors.

THEOREM 6.1. $\mathcal{C}_{1}(V) \oplus \mathcal{C}_{2}(V)=\left\{L \in \mathcal{K}_{C}(V)\right.$ with $\varphi_{L}$ constant $\}$.

Proof. This is clear from theorem 4.9 and theorem 3.5.

It follows that a $C$-curvature tensor with constant $\varphi$-holomorphic sectional curvature has vanishing Bochner component. 
Definition 6.2. A $C$-curvature tensor $L$ is $\eta$-Einsteman if there exist $a, b$ $\in \boldsymbol{R}$ such that

$$
\forall x, y \in V: L_{R}(x, y)=a g(x, y)+b \eta(x) \eta(y) .
$$

This is equivalent with $Q_{R}(L)=a I+b \eta \otimes \xi$ and $L$ is Einsteinian in the case $b=0$. Further we have

$$
L_{R}(\xi, \xi)=a+b=2 n \alpha .
$$

Using example 3 we get

COROLLARY 6.3. A C-curvature tensor with constant $\varphi$-holomorphic sectional curvature is $\eta$-Einsternian.

THEOREM 6.4. $\quad \mathcal{C}_{1}(V) \oplus \mathcal{C}_{2}(V) \oplus \mathcal{C}_{B}(V)=\left\{L \in \mathcal{K}_{C}(V) \mid L\right.$ is $\eta$-Einsterman $\}$.

Proof. Since $L_{B R}=0, \quad L$ is $\eta$-Einsteinian if $L \in \mathcal{C}_{1}(V) \oplus \mathcal{C}_{2}(V) \oplus \mathcal{C}_{B}(V)$ (use corollary 6.3).

Conversely, let $Q_{R}(L)=a I+b \eta \otimes \xi$. A straightforward computation shows that $L_{3}=0$.

COROLLARY 6.5. $L$ has constant $\varphi$-holomorphic sectronal curvature if and only if $L_{B}=0$ and $L$ is $\eta$-Einsternian.

We consider now again $C(\alpha)$-manifolds.

DEFINITION 6.6. A $C(\alpha)$-space form is a $C(\alpha)$-manifold with pointwise constant $\varphi$-holomorphic sectional curvature.

THEOREM 6.7. Let $M^{2 n+1}$ be a connected $C(\alpha)$-space form with $2 n+1 \geqq 5$. Then the $\varphi$-holomorphic sectional curvature is globally constant.

Proof. This can be done in exactly the same way as for Sasakian space forms (see [4]).

Co-Kähler space forms, which are $C(0)$-space forms, are considered in [14] and Kenmotsu space forms (which are $C(-1)$-space forms) in [12]. These last ones are automatically real space forms of constant sectional curvature -1 .

By defining $\eta$-Einstein almost $C(\alpha)$-manifolds pointwise using definition 6.2 we get from corollary 6.5 .

THEOREM 6.8. Let $M$ be a $C(\alpha)$-manifold. Then $M$ is a $C(\alpha)$-space form if and only if $M \eta$-Einsternian and $C$-Bochner flat.

This theorem can be compared with the well known theorems on conformally flat Einstein spaces and on Bochner flat Kähler-Einstein manifolds, which are respectively real and complex space forms. 
Finally we characterize $\mathcal{C}_{\bar{B}}^{\frac{1}{B}}(V)$.

Theorem 6.9. $L \in \mathcal{C}_{B}^{1}(V)$ (or $L$ is $C$-Bochner flat) if and only if $L=C_{A, I, a, \alpha}$ with $A \in \operatorname{Hom}^{\cos }(V)$ and $a, \alpha \in \boldsymbol{R}$.

Proof. Let $L \in C_{\bar{B}}^{1}(V)$. From $L_{B}=0$ we obtain $L=C_{A, I, a, \alpha}$ with

$$
A=\frac{1}{2(n+2)}\left(Q_{R}(L)-\frac{k}{2} I\right), \quad a=\frac{2 \alpha}{n+2} .
$$

The converse is obtained by straightforward computation.

\section{Norm inequalities.}

The decomposition gives rise to some interesting inequalities which can be used to characterize some special curvature tensors as in the Riemann, Kähler and quaternionic case $([1],[2],[7],[30])$. The use of these inequalities will be illustrated in a forthcoming paper. See also [35].

THEOREM 7.1. Let $L \in \mathcal{K}_{C}\left(V^{2 n+1}\right)$ and $L=\sum_{\imath=1}^{3} L_{\imath}+L_{B}$ with $L_{i} \in \mathcal{C}_{\imath}(V), L_{B} \in$ $\mathcal{C}_{B}(V)$. Then

$$
\begin{aligned}
\left\|L_{1}\right\|^{2} & =2 n(3 n+1) \alpha^{2}, \\
\left\|L_{2}\right\|^{2} & =\frac{2\{l(L)-n(3 n+1) \alpha\}^{2}}{n(n+1)}, \\
\left\|L_{3}\right\|^{2} & =\frac{4}{n+2}\left\{2\left\|Q_{R}(L)\right\|^{2}-8 n^{2} \alpha^{2}-\frac{1}{n}(l(L)-2 n \alpha)^{2}\right\}, \\
\left\|L_{B}\right\|^{2} & =\|L\|^{2}-\sum_{i=1}^{3}\left\|L_{i}\right\|^{2} .
\end{aligned}
$$

Proof. $\left\|L_{1}\right\|^{2}$ follows from (4.1). Further (4.2) implies

$$
g\left(C_{I, I}, \widetilde{L}\right)=16 l(\widetilde{L})
$$

for an arbitrary $\widetilde{L} \in C K(V)$. Using the formulas of example 3.8 and theorem 4.9 we get the formula for $\left\|L_{2}\right\|^{2}$.

Next, for $\widetilde{L} \in C K(V)$ we have

$$
g\left(\widetilde{L}, C_{Q_{R}(L), I}\right)=16 \sum_{\imath, \jmath=1}^{2 n} Q_{\imath j} \tilde{Q}_{\imath \jmath},
$$

where $\left(Q_{\imath j}\right)$ and $\left(\tilde{Q}_{\imath j}\right)$ are the matrices of $Q_{R}(L)$ resp. $Q_{R}(\tilde{L})$ with respect to an orthonormal $\varphi$-basis. Hence, using the formulas in examples 3.7 and 3.8, we obtain

$$
\begin{gathered}
\left\|C_{Q_{R}(L), I}\right\|^{2}=16\left\{2(n+2)\left(\left\|Q_{R}(L)\right\|^{2}-4 n^{2} \alpha^{2}\right)+(l(L)-2 n \alpha)^{2}\right\}, \\
g\left(C_{Q_{R}(L), I}, C_{I, I}\right)=64(n+1)(l(L)-2 n \alpha) .
\end{gathered}
$$


Then $\left\|L_{3}\right\|^{2}$ follows using theorem 4.9. $\left\|L_{B}\right\|^{2}$ is obtained by using the orthogonality of the decomposition.

Using theorem 6.1, theorem 6.4 and the relations $\|L\|^{2} \geqq\left\|L_{1}\right\|^{2}+\left\|L_{2}\right\|^{2},\left\|L_{3}\right\|^{2} \geqq 0$, we obtain

THEOREM 7.2. For all $L \in \mathcal{K}_{C(\alpha)}\left(V^{2 n+1}\right)$ we have

$$
\|L\|^{2} \geqq 2 n(3 n+1) \alpha^{2}+\frac{2(l(L)-n(3 n+1) \alpha)^{2}}{n(n+1)} .
$$

Equality holds if and only if $L$ has constant $\varphi$-holomorphic sectional curvature.

Theorem 7.3. For all $L \in \mathcal{K}_{C(\alpha)}\left(V^{2 n+1}\right)$ we have

$$
\left\|Q_{R}(L)\right\|^{2} \geqq 4 n^{2} \alpha^{2}+\frac{(l(L)-2 n \alpha)^{2}}{2 n} .
$$

Equality holds if and only if $L$ is $\eta$-Einsteman.

Since $\left\|L_{B}\right\|^{2} \geqq 0$, theorem 7.3 and corollary 6.5 imply

THEOREM 7.4. For all $L \in \mathcal{K}_{C(\alpha)}\left(V^{2 n+1}\right)$ we have

$$
\|L\|^{2} \geqq \frac{4}{n+1}\left\|Q_{R}(L)\right\|^{2}+\frac{4 \alpha(3 n-1)}{n+1}\{n(2 n+1) \alpha-l(L)\} .
$$

Equality holds if and only if $L$ has constant $\varphi$-holomorphic sectional curvature.

Remark that theorem 7.3 and theorem 7.4 imply theorem 7.2.

\section{Proper generalized $C(\alpha)$-curvature tensor fields on Sasakian manifolds.}

Definition 8.1. Let $(M, g, \varphi, \xi, \eta)$ be an almost co-Hermitian manifold and $L$ a $C^{\infty}(1,3)$-tensor field on $M . L$ is a (generalized) $C$-curvature tensor field on $M$ if for all $x \in M, L_{x}$ is a $C$-curvature tensor on $T_{x} M$. If $L_{x}$ has type $\alpha$ for all $x$, then $L$ is called a $C(\alpha)$-curvature tensor field on $M$.

Of course, the Riemann curvature tensor field $R$ on an almost $C(\alpha)$-manifold $M$ is a natural example of a $C(\alpha)$-curvature tensor field on $M$. Other examples can be obtained from examples 3.7 and 3.8 by taking $X, Y \in \mathscr{X}(M)$ instead of $x, y$ and $a \in \mathscr{F}(M)$ (a $C^{\infty}$ function on $M$ ), $A$ and $B C^{\infty}$ tensor fields of type $(1,1)$ so that for all $x \in M, A_{x}$ and $B_{x}$ are symmetric cocomplex linear maps of $T_{x} M$. We use for these examples also the same notations $C_{A, B, a, \alpha}, C_{A, B, \alpha}, C_{A, B}$ as in these examples 3.7 and 3.8.

For $R$ the second Branch identity plays an important role. So we want to consider $C$-curvature tensor fields satisfying this identity (see for example [17]). 
Definition 8.2. Let $\nabla$ denote the Riemannian connection on $M$. A $C$-curvature tensor field $L$ is called a proper field if and only if

$$
\forall X, Y, Z \in \mathfrak{X}(M):_{X, Y, Z}^{\subseteq}\left(\nabla_{X} L\right)(Y, Z)=0 .
$$

Proper C-curvature fields are special Riemannian double forms on $M$ (see [8]).

We give an example for Sasakıan manıfolds. We consider the $C(\alpha)$-curvature tensor field $E$ on $M$ given by

$$
E(X, Y)=s \wedge(X, Y)+(s-\alpha)\{\wedge(\varphi X, \varphi Y)+\mathscr{H}(X, Y)+2 g(X, \varphi Y) \varphi\},
$$

where $s \in \mathscr{F}(M)$. Then we have

ExAMPLE. 8.3. Let $M^{2 n+1}, n>1$, be a connected Sasakzan manrfold. Then $E$ us proper if and only if $s$ is a constant function on $M$.

Proof. Suppose $E$ is proper. Then the statement is a direct generalization of Schur's lemma for the Riemann curvature tensor field on Sasakian manifolds (see [4]).

The converse is a straightforward calculation using

$$
\left\{\begin{array}{l}
\left(\nabla_{X} \varphi\right) Y=g(X, Y) \xi-\eta(Y) X, \\
\nabla_{X} \xi=-\varphi X,
\end{array}\right.
$$

which are well known relations on Sasakian manifolds (see theorem 2.6).

In what follows we suppose always that $M$ is a Sasakian manifold and we consider a generalized $C(\alpha)$-curvature tensor field on $M$. By using pointwise the decomposition theorem we obtain four $C$-curvature tensor fields $L_{1}, L_{2}, L_{3}$ and $L_{B}$ on $M$. A natural question arises now: if $L$ is a proper field, does then the same property holds for $L_{\imath}(i=1,2,3)$ and $L_{B}$ ? As we can see from [16], [17], the Codazzi equation for $Q_{R}(L)$, i. e.

$$
\forall X, Y \in \mathscr{X}(M):\left(\nabla_{X} Q_{R}(L)\right) Y=\left(\nabla_{Y} Q_{R}(L)\right) X,
$$

will play an important role. This natural condition (see [9]) has very strong consequences for $C(\alpha)$-curvature tensor fields on Sasakian manifolds. We study this now more in detail.

THEOREM 8.4. Let $L$ be a $C(\alpha)$-curvature tensor field on a Sasakian manifold $M^{2 n+1}$. If the Riccr tensor field $Q_{R}(L)=Q$ satısfies the Codazzi equation, then

$$
Q_{R}(L)=2 n \alpha I \text {. }
$$

To prove this we need a generalization to almost co-Hermitian manifolds of a theorem of Tanno [23] concerning hybrid tensor fields on Kähler manifolds. 
Definition 8.5. Let $M$ be an almost co-Hermitian manifold. A $(0, r)$-tensor field $T$ on $M$ is called $C$-hybrid with respect to $\imath$ and $\jmath$ if

$$
\begin{aligned}
\forall X_{1}, \cdots, X_{r} \in \mathscr{X}(M): T\left(X_{1}, \cdots, X_{\imath}, \cdots, X_{\jmath}, \cdots, X_{r}\right) \\
=T\left(X_{1}, \cdots, \varphi X_{\imath}, \cdots, \varphi X_{\jmath}, \cdots, X_{r}\right) .
\end{aligned}
$$

COROLlary 8.6. Let $T$ be a C-hybrid tensor with respect to 2 and J. Then

$$
T\left(X_{1}, \cdots, \underset{\uparrow}{\xi}, \cdots, X_{\jmath}, \cdots, X_{r}\right)=T\left(X_{1}, \cdots, X_{\imath}, \cdots, \underset{\uparrow}{\xi}, \cdots, X_{r}\right)=0 .
$$

THEOREM 8.7. Let $T$ be a $(0, r)$-tensor field with $r \geqq 3$ and which is $C$-hybrid with respect to $(\imath, j),(\jmath, k)$ and $(i, k)$. Then $T=0$.

Proof. First we take $X_{i} \in \mathfrak{X}(M)$ such that $\eta\left(X_{\imath}\right)=0$ for all $1 \leqq \imath \leqq r$. Then we have $\varphi^{2} X_{\imath}=-X_{\imath}$ and

$$
\begin{aligned}
& T\left(X_{1}, \cdots, X_{\imath}, \cdots, X_{\jmath}, \cdots, X_{k}, \cdots, X_{r}\right) \\
& \quad=T\left(X_{1}, \cdots, \varphi X_{\imath}, \cdots, \varphi X_{\jmath}, \cdots, X_{k}, \cdots, X_{r}\right) \\
& \quad=T\left(X_{1}, \cdots, \varphi X_{\imath}, \cdots, \varphi^{2} X_{\jmath}, \cdots, \varphi X_{k}, \cdots, X_{r}\right) \\
& \quad=T\left(X_{1}, \cdots, \varphi^{2} X_{\imath}, \cdots, \varphi^{2} X_{\jmath}, \cdots, \varphi^{2} X_{k}, \cdots, X_{r}\right) \\
& \quad=-T\left(X_{1}, \cdots, X_{\imath}, \cdots, X_{\jmath}, \cdots, X_{k}, \cdots, X_{r}\right) .
\end{aligned}
$$

Next we take $X_{\imath}$ arbitrary. Then the theorem follows at once using corollary 8.6 and by linearity since $\left(X_{\imath}\right)_{x}=a_{i}\left(Y_{\imath}\right)_{x}+b_{i} \xi_{x}$ where $\eta\left(Y_{\imath}\right)=0$.

THEOREM 8.8. Let $L$ be a $C(\alpha)$-curvature tensor field on a Sasakian manrfold. If $Q_{R}(L)=Q$ satisfies the Codazzi equation, then $Q$ is parallel, i.e. $\nabla Q=0$.

Proof. Let $S$ denote the Ricci tensor field of type $(0,2)$ associated with $L$, i. e. $S(X, Y)=g(Q X, Y)$. First the Codazzi equation implies

$$
\forall X, Y, Z \in \mathscr{X}(M):\left(\nabla_{X} S\right)(Y, Z)=\left(\nabla_{Y} S\right)(X, Z) .
$$

Further, since $Q$ is cocomplex linear we have, using theorem 3.6, for all $X, Y \in$ $\mathscr{X}(M)$ :

In particular we get

$$
\begin{aligned}
S(\varphi X, \varphi Y) & =g(Q \varphi X, \varphi Y)=g(\varphi Q X, \varphi Y) \\
& =g(Q X, Y)-\eta(Q X) \eta(Y) \\
& =S(X, Y)-2 n \alpha \eta(X) \eta(Y) .
\end{aligned}
$$

$$
S(\xi, \xi)=2 n \alpha .
$$

Next we show that the $(0,3)$-tensor field $\nabla S$ is $C$-hybrid in the last two arguments, i. e. $\left(\nabla_{X} S\right)(Y, Z)=\left(\nabla_{X} S\right)(\varphi Y, \varphi Z)$. Using (8.2) it follows then at once 
that $\nabla S$ is completely $C$-hybrid and hence, by theorem 8.7, we obtain the required result.

We start the proof by taking $Y, Z \in\{\xi\}^{\perp}$. Then

$$
\begin{aligned}
\left(\nabla_{X} S\right)(\varphi Y, \varphi Z)= & X\{S(\varphi Y, \varphi Z)\}-S\left(\nabla_{X}(\varphi Y), Z\right)-S\left(\varphi Y, \nabla_{X}(\varphi Z)\right) \\
= & X\{S(Y, Z)\}-S\left(g(X, Y) \xi+\varphi \nabla_{X} Y, \varphi Z\right) \\
& -S\left(\varphi Y, g(X, Z) \xi+\varphi \nabla_{X} Z\right)
\end{aligned}
$$

by (8.1) and (8.3). Since $Q \xi=2 n \alpha \xi$ (theorem 3.6) we have $S(\xi, \varphi Z)=S(\xi, \varphi Y)=0$ and hence

$$
\left(\nabla_{X} S\right)(\varphi Y, \varphi Z)=X\{S(Y, Z)\}-S\left(\varphi \nabla_{X} Y, \varphi Z\right)-S\left(\varphi Y, \varphi \nabla_{X} Z\right)=\left(\nabla_{X} S\right)(Y, Z),
$$

using again (8.3).

Further, let $X, Y \perp \xi$. Then with (8.1) we get

But (8.2) implies

$$
\left(\nabla_{X} S\right)(Y, \xi)=S(Y, \varphi X)-2 n \alpha g\left(\nabla_{X} Y, \xi\right) .
$$

$$
\left(\nabla_{X} S\right)(Y, \xi)=S(X, \varphi Y)-2 n \alpha g\left(\nabla_{Y} X, \xi\right)
$$

and, since $\xi$ is Killing, we get by adding the two relations

Next

$$
\left(\nabla_{X} S\right)(Y, \xi)=0 \text {. }
$$

$$
\left(\nabla_{X} S\right)(\xi, \xi)=0 \text { for all } X \in \mathfrak{X}(M) \text {, }
$$

since we have $\nabla_{X} \xi=-\varphi X$ and $S(\varphi X, \xi)=0$ (theorem 3.6). The theorem follows now at once using the Codazzi equation since

$$
\left(\nabla_{X} S\right)(Y, \xi)=0=\left(\nabla_{X} S\right)(\varphi Y, \varphi \xi) \text { for } X, Y \in \mathscr{X}(M) .
$$

THEOREM 8.9. Let $L$ be a $C(\alpha)$-curvature tensor field on $M$ with parallel Riccr tensor $Q=Q_{R}(L)$. Then

$$
Q_{R}(L)=2 n \alpha I .
$$

Proof. Since $Q$ is parallel and cocomplex linear we get from $\left(\nabla_{X} Q\right) \varphi Y=0$ :

On the other hand

$$
\begin{aligned}
\nabla_{X}(\varphi Q Y)-\varphi Q \nabla_{X} Y & =Q\left(\nabla_{X} \varphi\right) Y \\
& =Q\{g(X, Y) \xi-\eta(Y) X\} \\
& =2 n \alpha g(X, Y) \xi-\eta(Y) Q X .
\end{aligned}
$$

$$
\nabla_{X}(\varphi Q Y)-\varphi Q \nabla_{X} Y=\left(\nabla_{X} \varphi\right) Q Y=g(X, Q Y) \xi-2 n \alpha \eta(Y) X .
$$

Combining the two relations and putting $Y=\xi$ we obtain the required result.

Remark that theorem 8.9 is proved in a different way for the Riemann tensor 
in [18]. The result above follows also from the fact that a Sasakian manifold is irreducible $\left[23^{\prime}\right]$ and from $S(\xi, \xi)=2 n \alpha$.

Proof of theorem 8.4. Combine theorems 8.8 and 8.9 .

Further we have from theorem 8.4 (or from the fact that a Sasakian manifold is irreducible)

Corollary 8.10. Let $M$ be a Sasakian manifold with parallel Rucci tensor. Then $M$ is Einsterman.

This corollary is important in view of the classes studied in [9].

Combining theorem 8.4 and example 8.3 we obtain

TheOREM 8.11. Let $M^{2 n+1}, n>1$, be a connected Sasakian manrfold and $L$ a $C(\alpha)$-curvature tensor field on $M$ whose Riccr tensor $Q_{R}(L)$ satisfies the Codazzl equation. Then $C_{Q_{R}(L), I}$ is a proper $C(\alpha)$-curvature tensor field.

Now we return to our main problem. First we remark that by example 8.3 and because $\alpha$ is constant, the component $L_{1}$ is always proper.

THEOREM 8.12. Let $L$ be a (generalized) $C(\alpha)$-curvature tensor field on a connected Sasakran manifold $M^{2 n+1}, n>1$, and let

$$
L=\sum_{i=1}^{3} L_{i}+L_{B}
$$

be the natural decomposition. If $L$ is proper and $Q_{R}(L)$ satisfies the Codazzi equation, then $L_{2}, L_{3}$ and $L_{B}$ are also proper. Conversely, if $L_{2}, L_{3}$ and $L_{B}$ are proper, then $Q_{R}(L)$ satisfies the Codazzi equation.

Proof. If $L$ is proper, the theorem follows at once from example 8.3, theorem 8.4 and theorem 4.9 since theorem 8.4 implies that the scalar curvature $l(L)$ is constant.

Conversely, since $L_{2}$ is proper, $k$ and so $l(L)$ is constant by example 8.3. Further, since $L_{3}$ is proper, $C_{Q_{R}(L), I}$ has to be proper and so

$$
\underset{X, Y, Z}{\mathfrak{S}}\left(\nabla_{X} C_{Q_{R}(L), I}\right)(Y, Z) U=0 .
$$

Using

$$
\begin{gathered}
\left(\nabla_{X}(\varphi Q)\right) Y=\left(\nabla_{X} Q\right) \varphi Y+2 n \alpha g(X, Y) \xi-\eta(Y) Q X, \\
\eta\left\{\left(\nabla_{X} Q\right) Y-\left(\nabla_{Y} Q\right) X\right\}=2 g(X, \varphi Y)-2 n \alpha\left\{\eta\left(\nabla_{X} Y\right)-\eta\left(\nabla_{Y} X\right)\right\} \\
=2 g(\varphi X, \varphi Y)-4 n \alpha g(Y, \varphi X),
\end{gathered}
$$

which are easily verified, we compute the cyclic sum over $X, Y, Z$ of

$$
g\left(\left(\nabla_{X} C_{Q_{R}(L), I}\right)(Y, Z) U, \xi\right) \text { for } X, Y, Z, U \perp \xi \text {. }
$$


This gives the condition

$$
\begin{aligned}
& g(U, \varphi Z) S(X, Y)-2 n \alpha g(U, \varphi Z) g(X, Y)+g(U, \varphi X) S(Y, Z) \\
& \quad-2 n \alpha g(U, \varphi X) g(Z, Y)+g(U, \varphi Y) S(X, Z)-2 n \alpha g(U, \varphi Y) g(X, Z) \\
& \quad-2 n \alpha g(X, U) g(Z, \varphi Y)-2 n \alpha g(Y, U) g(X, \varphi Z)-2 n \alpha g(Z, U) g(\varphi X, Y)=0 .
\end{aligned}
$$

For $X=Y=Z$ and $g(X, X)=1$ this reduces to

$$
g(U, \varphi X) S(X, X)-2 n \alpha g(U, \varphi X)=0
$$

and, since $U \perp \xi$ is arbitrary, we get

$$
S(X, X) \varphi X=2 n \alpha \varphi X .
$$

Hence $S(X, X)=2 n \alpha$. This implies with $Q \xi=\ln \alpha \xi$ that $Q=2 n \alpha I$ and for Sasakian manifolds this is equivalent with the Codazzi equation.

COROLLARY 8.13. Let $M^{2 n+1}, n>1$, be a connected Sasakran manrold and $L$ a proper $C(\alpha)$-curvature tensor field with constant scalar curvature $i(L)$. Then $L_{B}$ is proper if and only if $Q_{R}(L)$ satisfies the Codazzi equation.

These theorems are analogous with those for the Weyl and Bochner tensor proved in [16], [17]. This is also true for the following considerations.

Let $U_{C}(M)$ be the vector space of all $C^{\infty}$ tensor fields $A$ of type $(1,1)$ on a Sasakian manifold $M^{2 n+1}$, satisfying the following properties:

(i) $A$ is symmetric in each point;

(ii) $A$ is cocomplex linear in each point with $A \xi=\beta \xi$, where $\beta$ is constant on $M$;

(iii) $A$ satisfies the Codazzi equation $\left(\nabla_{X} A\right) Y=\left(\nabla_{Y} A\right) X$;

(iv) Trace $A$ is constant on $M$.

Just as in theorem 8.4 one proves that on a Sasakian manifold

$$
\mathcal{U}_{C}=\{\lambda I \mid \lambda \in \boldsymbol{R}\} .
$$

On the other hand, let $\mathcal{L}_{C}(M)$ be the vector space of all proper generalized $C$-curvature tensor fields of constant type on $M$ such that their Ricci tensor satisfies the Codazzi equation. Then the map

defined by

$$
\mathcal{A}: \vartheta_{C}(M) \rightarrow \mathcal{L}_{C}(M) ; A \mapsto L_{A}
$$

$$
\begin{aligned}
L_{A}(X, Y)= & \frac{1}{2(n+2)} C_{A, I}(X, Y)-\frac{k}{4(n+2)} C_{I, I}(X, Y) \\
& -\frac{\alpha}{n+2}\left\{-2 \wedge(X, Y)+n\left(\wedge(\varphi X, \varphi Y)+\mathcal{H}(X, Y)+2 g(X, \circlearrowleft Y)_{\bullet}\right)\right\}
\end{aligned}
$$


where $k=\frac{\operatorname{tr} A+2 n \alpha}{2(n+1)}$ and $A \xi=2 n \alpha \xi$, is a linear map. $L_{A} \in \mathcal{L}_{C}(M)$ because $Q_{R}\left(L_{A}\right)=A$ and $\left(L_{A}\right)_{B}=0$. Further, the map $\mathcal{A}$ is in jective since $L_{A}=L_{A^{\prime}}$ implies $Q_{R}\left(L_{A}\right)=Q_{R}\left(L_{A^{\prime}}\right)$ or $A=A^{\prime}$.

THEOREM 8.14. Let $M^{2 n+1}$ be a $(2 n+1)$-dimensional Sasakian manifold. If $n=1, \mathcal{A}$ is an isomorphism. Otherwise $\mathcal{A}$ is an isomorphism on the subspace

$$
\left\{L \in \mathcal{L}_{C}(M) \mid L_{B}=0\right\} .
$$

Proof. For $n=1$ we obtain

$$
L(X, Y)=\left(L_{1}+L_{2}\right)(X, Y)=\frac{\alpha}{2}\{\wedge(X, Y)-\wedge(\varphi X, \varphi Y)-\mathscr{H}(X, Y)-2 g(X, \varphi Y) \varphi\} .
$$

So $\mathcal{L}_{C}(M)$ is 1 -dimensional. If $n \geqq 2$ we have for $L \in \mathcal{L}_{C}(M)$ with $L_{B}=0$ :

$$
\begin{aligned}
L(X, Y)= & \frac{1}{2(n+2)} C_{Q_{R}(L), I}(X, Y)-\frac{k}{4(n+2)} C_{I, I}(X, Y) \\
& -\frac{\alpha}{n+2}\{-2 \wedge(X, Y)+n(\wedge(\varphi X, \varphi Y)+\mathscr{H}(X, Y)+2 g(X, \varphi Y) \varphi\}
\end{aligned}
$$

and this implies $L=L_{Q_{R}(L)}$. So the map is surjective on this subspace.

\section{REFERENCES}

[1-: M. Berger, Le spectre des variétés riemanniennes, Rev. Roumaine Math. Pures Appl., 23 (1968), 915-931.

[2] M. BERger, P. GAUduchon \& E. MAzet, Le spectre d'une variété riemannienne, Lecture Notes in Mathematics, vol. 194, Springer Verlag, Berlin and New York, 1971.

[3] D. BlaIR, The theory of quasi-Sasakian structures, J. Differential Geometry, 1 (1967), 331-345.

[4] D. BlaIR, Contact manifolds in Riemannian geometry, Lecture Notes in Mathematics, vol. 509, Springer Verlag, Berlin and New York, 1976.

[5] R. L. Bishop \& B. O'Neill, Manifolds of negative curvature, Trans. Amer. Math. Soc., 145 (1969), 1-49.

[6] J. Bouzon, Structures presque-cocomplexes, Rend. Sem. Mat. Univ. e Politec. Torino, 24 (1964-65), 53-123.

[7] H. Donelly, Topology and Einstein Kähler metrics, J. Differential Geometry, 11 (1976), 259-264.

[8] A. GRAY, Some relations between curvature and characteristic classes, Math. Ann., 184 (1970), 257-267.

[9] A. Gray, Einstein-like manifolds which are not Einstein, Geometraae Dedicata, 7 (1978), 259-280.

[10] A. Gray \& L. Hervella, The sixteen classes of almost Hermitian manifolds and their linear invariants, Ann. Mat. Pura Appl., 123 (1980), 35-58.

[11] D. Janssens, Krommingstensoren, Decompositietheorie en toepassingen, Doctoraatsverhandeling, Kath. Unıv. Leuven, 1977.

[12] K. Kenmotsu, A class of almost contact Riemannian manifolds, Tôhoku Math. J., 24 (1972), 93-103.

[13] R.S. Kulkarni, On the Bianchi identities, Math. Ann., 199 (1972), 175-204.

[14] G.D. LuDDEN, Submanifolds of cosymplectic manifolds, J. Differential Geometry, 
4 (1970), 237-244.

[15] M. Matsumoto \& G. ChĒman, On the C-Bochner curvature tensor, TRU Math., 5 (1969), 21-30.

[16] H. MoRI, On the decomposition of generalized $K$-curvature tensor fields, Tôhoku Math. J., 25 (1973), 225-235.

[17] K. Nomizu, On the decomposition of generalized curvature tensor fields, Differential Geometry, Kinokuniya, Tokyo, 1972, 335-345.

[18] M. Okumura, Some remarks on space with a certain contact structure, Tôhoku Math. J., 14 (1962), 135-145.

[19] S. SASAKI, Almost contact manifolds I, II, III, Lecture Notes, Math. Institute Tôhoku Unıv., 1965, 1967, 1968.

[20] I. M. Singer \& J. A. Thorpe, The curvature of 4-dimensional Einstein spaces, Global Analysıs (papers in honor of K. Kodaira), Univ. of Tokyo Press, Tokyo, 1969, 355-365.

[21] M. Sitaramayya, Curvature tensors in Kähler manifolds, Trans. Amer. Math. Soc., 183 (1973), 341-353.

[22] S. TANno, The automorphism groups of almost contact Riemannian manifolds, Tôhoku Math. J., 21 (1969), 21-38.

[23] S. TAnno, 4-dimensional conformally flat Kähler manifolds, Tôhoku Math. J., 24 (1972), 501-504.

[23'] S. Tanno, Locally symmetric $K$-contact Riemannian manifolds, Proc. Japan Acad., 49 (1967), 581-583.

[24] L. Vanhecke, The Bochner curvature tensor on almost Hermitian manifolds, Rend. Sem. Mat. Univ. e Politec. Tornno, 34 (1975-76), 21-38.

[25] L. VANHECKE, Almost Hermitian manifolds with $J$-invariant Riemannian curvature tensor, Rend. Sem. Mat. Unvv. e Politec. Torino, 34 (1975-76), 487-498.

[26] L. VAnHECKE, Antiholomorphic submanifolds of generalized complex space forms, Bull. Inst. Math. Acad. Sinica, 4 (1976), 127-140.

[27] L. VANHECKE, On the decomposition of curvature tensor fields on almost Hermitian manifolds, Conf. on Diff. Geometry, Proc., Michigan State Unıv., 1976, 16-33.

[28] L. VANHECKE, The Bochner curvature tensor on almost Hermitian manifolds, Geometrae Dedicata, 6 (1977), 389-397.

[29] L. Vanhecke \& F. Bouten, Constant type for almost Hermitian manifolds, Bull. Math. Soc. Math. R.S. Roumanie, 20 (1976), 415-422.

[30] Y. WATANABE, On the characteristic functions of harmonic quaternion Kählerian spaces, Kōdai Math. Sem. Rep., 27 (1976), 410-420.

[31] H. WeYL, The classical groups. Their invariants and representations, Princeton Univ. Press, Princeton, 1946.

[32] K. Yano \& M. Kon, Antı-invarıant submanifolds, Marcel Dekker, New York, 1976.

[33] Y. Yoshimatsu, On the decomposition of generalized S-curvature-like tensor fields, Tôhoku Math. J., 29 (1977), 235-254.

[34] F. TRICERRi \& L. VAnHecke, Curvature tensors on almost Hermitian manifolds, to appear in Trans. Amer. Math. Soc.

[35] Y Shibuya, The Spectrum of Sasakian manifolds, Kodai Math. J., 3 (1980), $197-211$

Department of Mathematics

Katholieke Universiteit LeUven

CelestijnenlaAn 200B

B-3030 Leuven (BELgium) 